\title{
On absolute central moments of Poisson distribution
}

\author{
Pavel S. Ruzankin*1,2 \\ ${ }^{1}$ Sobolev Institute of Mathematics, Novosibirsk, Russia \\ ${ }^{2}$ Novosibirsk State University, Novosibirsk, Russia
}

\begin{abstract}
A recurrence formula for absolute central moments of Poisson distribution is suggested. Keywords: Poisson distribution, absolute central moment.
\end{abstract}

Let $X$ be a Poisson random variable with mean $m$. In this paper, we study absolute central moments $\mathbf{E}|X-a|^{r}$ about $a$ for naturals $r$. Explicit representations for such moments may be useful in situations where we want to test whether observations in a large sample are independent Poisson with given means, which is the case, for instance, for some image reconstruction techniques in emission tomography (e.g., see Ben Bouallègue et al., 2013 and Hebert, 1990).

An explicit representation for the mean deviation was obtained independently by Crow (1958) and Ramasubban (1958):

$$
\mathbf{E}|X-m|=2 e^{-m} \frac{m^{\lfloor m\rfloor+1}}{\lfloor m\rfloor !},
$$

where $\lfloor\cdot\rfloor$ denotes the floor function.

Kendall (1943, relations (5.21) and (5.22) on p. 121) has showed that, for all integers $r \geq 2$,

$$
\begin{aligned}
\mathbf{E}(X-m)^{r} & =m \sum_{k=0}^{r-2}\left(\begin{array}{c}
r-1 \\
k
\end{array}\right) \mathbf{E}(X-m)^{k}, \\
\mathbf{E}(X-m)^{r+1} & =r m \mathbf{E}(X-m)^{r-1}+m \frac{d}{d m} \mathbf{E}(X-m)^{r} .
\end{aligned}
$$

Denote by ${ }_{1} F_{1}$ the confluent hypergeometric function of the first kind

$$
{ }_{1} F_{1}(\alpha, \beta, z)=\sum_{n=0}^{\infty} \frac{z^{n}}{n !} \prod_{j=0}^{n-1} \frac{\alpha+j}{\beta+j},
$$

*email: ruzankin@math.nsc.ru 
where $\prod_{j=0}^{-1}=1$. Katti (1960) has derived the following representation for absolute central moments of $X$ about $a$ for all odd $r \geq 1$ :

$$
\mathbf{E}|X-a|^{r}=-\mathbf{E}(X-a)^{r}+\frac{e^{-m} m^{\lfloor a\rfloor+1}}{(\lfloor a\rfloor+1) !} G^{(r)}(0,0),
$$

where

$$
G(\beta, t)=\exp \{t(\lfloor a\rfloor-a+\beta+1)\}_{1} F_{1}\left(\beta+1, \beta+\lfloor a\rfloor+2, m e^{t}\right),
$$

$G^{(r)}(\beta, t)$ is its $r$-th partial derivative with respect to $t$, which, for $t=0$, can be computed by the recurrence formula

$$
G^{(s+1)}(\beta, 0)=(\lfloor a\rfloor-a+\beta+1) G^{(s)}(\beta, 0)+\frac{m(\beta+1)}{\beta+\lfloor a\rfloor+2} G^{(s)}(\beta+1,0)
$$

for $s=1,2, \ldots$, where

$$
G^{(0)}(\beta, 0):=G(\beta, 0)={ }_{1} F_{1}(\beta+1, \beta+\lfloor a\rfloor+2, m) .
$$

The main goal of this paper is to suggest a simpler recurrence formula for absolute central moments of $X$ about $a$.

Define the sign function

$$
\operatorname{sign}(y)= \begin{cases}-1 & \text { if } y \leq 0 \\ 1 & \text { if } y>0\end{cases}
$$

and put

$$
\begin{aligned}
F(b) & :=\mathbf{P}(X \leq b) \\
C(r, a) & :=\mathbf{E}(X-a)^{r} \\
D(r, a, b) & :=\mathbf{E}(X-a)^{r} \operatorname{sign}(X-b), \\
B(r, a, f) & :=\mathbf{E}(X-a)^{r} f(X),
\end{aligned}
$$

where $0^{0}=1$ by definition, $f$ is a real-valued function. Here $F(b)$ is the cumulative distribution function of $X$, and $C(r, a)$ is the $r$-th central moment about $a$. The above definition of the sign function is not common for $y=0$, but is chosen here for the sake of convenience, to provide the equality

$$
D(0, a, b)=1-2 F(b) .
$$

We have

$$
\mathbf{E}|X-a|^{r}= \begin{cases}C(r, a) & \text { if } r \text { is even } \\ D(r, a, a) & \text { if } r \text { is odd }\end{cases}
$$


Theorem 1. For all integers $r \geq 1$, reals $b \geq 0$ and $a$, and functions $f$ such that $\mathbf{E} X^{r}|f(X)|<\infty$, the following recurrence relations are valid:

$$
\begin{aligned}
C(r, a)= & (m-a) C(r-1, a)+m \sum_{k=0}^{r-2}\left(\begin{array}{c}
r-1 \\
k
\end{array}\right) C(k, a) \\
= & m C(r-1, a-1)-a C(r-1, a), \\
D(r, a, b)= & (m-a) D(r-1, a, b)+m \sum_{k=0}^{r-2}\left(\begin{array}{c}
r-1 \\
k
\end{array}\right) D(k, a, b) \\
& +2(\lfloor b\rfloor+1-a)^{r-1} e^{-m} \frac{m^{\lfloor b\rfloor+1}}{\lfloor b\rfloor !} \\
B(r, a, f)= & (m D(r-a) B(r-1, a-1, b-1)-a D(r-1, a, b), \\
& \quad+m \sum_{k=0}^{r-1}\left(\begin{array}{c}
r-1 \\
k
\end{array}\right) B(k, a, \Delta f),
\end{aligned}
$$

where $0^{0}=1$ by definition,

$$
\Delta f(j):=f(j+1)-f(j),
$$

and all the expectations $B$ are finite.

Remark 1. The same approach can be used to obtain similar relations for binomial and hypergeometric distributions.

The following statement is a direct consequence of relations (7) and (4).

\section{Corollary 1.}

$$
\begin{aligned}
\mathbf{E}|X-m|^{3}= & m(1-2 F(m))+2\left((m-\lfloor m\rfloor)^{2}+2\lfloor m\rfloor+1\right) e^{-m} \frac{m^{\lfloor m\rfloor+1}}{\lfloor m\rfloor !} \\
\mathbf{E}|X-m|^{5}= & \left(10 m^{2}+m\right)(1-2 F(m))+2\left((\lfloor m\rfloor+1-m)^{4}\right. \\
& \left.+2 m\left(2(m-\lfloor m\rfloor)^{2}+7\lfloor m\rfloor+7-3 m\right)\right) e^{-m} \frac{m^{\lfloor m\rfloor+1}}{\lfloor m\rfloor !}
\end{aligned}
$$

Notice that the cumulative distribution function $F$ can be calculated, e.g., by the built-in function ppois() in $\mathrm{R}$ or by the function scipy.stats.poisson.cdf() in Python. 
Proof of Theorem 1. First, let us prove (7). By Proposition 1 in Borisov and Ruzankin (2002, p. 1660), we have the equivalences

$$
\begin{aligned}
\mathbf{E}\left|(X-a)^{r} f(X)\right|<\infty & \Leftrightarrow \mathbf{E} X^{r}|f(X)|<\infty \Leftrightarrow \mathbf{E}\left|\Delta^{r} f(X)\right|<\infty \\
& \Leftrightarrow \mathbf{E} X^{r-1}|\Delta f(X)|<\infty \Leftrightarrow \mathbf{E}\left|(X-a)^{r-1} \Delta f(X)\right|<\infty,
\end{aligned}
$$

where the $\Leftrightarrow$ sign means "if and only if", $\Delta^{r}$ means applying the $\Delta$ operator $r$ times. Hence, all the expectations $B$ in (7) are finite. For $r \geq 1$, we have

$$
\begin{aligned}
& e^{m} B(r, a, f)=\sum_{j=0}^{\infty} \frac{(j-a)^{r} f(j) m^{j}}{j !}=\sum_{j=0}^{\infty} \sum_{k=0}^{r}\left(\begin{array}{l}
r \\
k
\end{array}\right) \frac{j^{k}(-a)^{r-k} f(j) m^{j}}{j !} \\
= & \sum_{j=0}^{\infty} \sum_{k=1}^{r}\left(\begin{array}{c}
r-1 \\
k-1
\end{array}\right) \frac{j^{k}(-a)^{r-k} f(j) m^{j}}{j !}+\sum_{j=0}^{\infty} \sum_{k=0}^{r-1}\left(\begin{array}{c}
r-1 \\
k
\end{array}\right) \frac{j^{k}(-a)^{r-k} f(j) m^{j}}{j !} \\
& =\sum_{j=0}^{\infty} \frac{j(j-a)^{r-1} f(j) m^{j}}{j !}-\sum_{j=0}^{\infty} \frac{a(j-a)^{r-1} f(j) m^{j}}{j !} \\
= & \sum_{j=0}^{\infty} \frac{(j+1-a)^{r-1} f(j+1) m^{j+1}}{j !}-a e^{m} B(r-1, a, f) \\
= & \sum_{j=0}^{\infty} \frac{(j+1-a)^{r-1}(f(j)+\Delta f(j)) m^{j+1}}{j !}-a e^{m} B(r-1, a, f) \\
= & m \sum_{k=0}^{r-1}\left(\begin{array}{c}
r-1 \\
k
\end{array}\right) \sum_{j=0}^{\infty} \frac{(j-a)^{k}(f(j)+\Delta f(j)) m^{j}}{j !}-a e^{m} B(r-1, a, b) \\
= & m \sum_{k=0}^{r-1}\left(\begin{array}{c}
r-1 \\
k
\end{array}\right) e^{m}(B(k, a, f)+B(k, a, \Delta f))-a e^{m} B(r-1, a, b),
\end{aligned}
$$

which proves (7).

Relation (5) follows immediately from (9) with $f(j) \equiv 1$. Analogously, (10) implies (6).

Let us now prove (7). By (10), for $r \geq 1$, we have

$$
e^{m} D(r, a, b)=\sum_{j=0}^{\infty} \frac{(j+1-a)^{r-1} \operatorname{sign}(j+1-b) m^{j+1}}{j !}-a e^{m} D(r-1, a, b)
$$


which proves (8) . Notice that $\operatorname{sign}(j+1-b) \neq \operatorname{sign}(j-b)$ for $j=\lfloor b\rfloor$ only. Hence, (11) equals

$$
\begin{aligned}
& =\sum_{j=0}^{\infty} \frac{(j+1-a)^{r-1} \operatorname{sign}(j-b) m^{j+1}}{j !}+\frac{2(\lfloor b\rfloor+1-a)^{r-1} m^{\lfloor b\rfloor+1}}{\lfloor b\rfloor !}-a e^{m} D(r-1, a, b) \\
& =m \sum_{k=0}^{r-1}\left(\begin{array}{c}
r-1 \\
k
\end{array}\right) \sum_{j=0}^{\infty} \frac{(j-a)^{k} \operatorname{sign}(j-b) m^{j}}{j !}+\frac{2(\lfloor b\rfloor+1-a)^{r-1} m^{\lfloor b\rfloor+1}}{\lfloor b\rfloor !}-a e^{m} D(r-1, a, b) \\
& =m \sum_{k=0}^{r-1}\left(\begin{array}{c}
r-1 \\
k
\end{array}\right) e^{m} D(k, a, b)+\frac{2(\lfloor b\rfloor+1-a)^{r-1} m^{\lfloor b\rfloor+1}}{\lfloor b\rfloor !}-a e^{m} D(r-1, a, b),
\end{aligned}
$$

which proves (7).

The theorem is proved.

\section{References}

F. Ben Bouallègue, J.F. Crouzet, D. Mariano-Goulart. 2013. A heuristic statistical stopping rule for iterative reconstruction in emission tomography. Ann Nucl Med, 27:84-95.

I.S. Borisov, P.S. Ruzankin. 2002. Poisson approximation for expectations of unbounded functions of independent random variables. Ann Probab, 30(4):1657-1680.

E.L. Crow. 1958. The Mean Deviation of the Poisson Distribution. Biometrika, 45(3/4):556559.

T.J. Hebert. 1990. Statistical stopping criteria for iterative maximum likelihood reconstruction of emission images. Phys Med Biol, 35:1221-1232.

S.K. Katti. 1960. Moments of the Absolute Difference and Absolute Deviation of Discrete Distributions. Ann Math Stat, 31(1):78-85.

M.G. Kendall. 1943. The Advanced Theory of Statistics, Vol. 1, London: Griffin.

T.A. Ramasubban. 1958. The Mean Difference and the Mean Deviation of Some Discontinuous Distributions. Biometrika, 45(3/4):549-556. 